\title{
In situ Methods for Identifying Reactive Surface Intermediates during Hydrogenolysis Reactions: C-O Bond Cleavage on Nanoparticles of Nickel and Nickel Phosphides
}

Megan E. Witzke, $\uparrow$ Abdulrahman Almithn, $\S$ Christian L. Conrad, $\uparrow$ Mark D. Triezenberg, $\uparrow$ David D. Hibbitts, $\S$, and David W. Flaherty $\dagger, *$

†Department of Chemical and Biomolecular Engineering, University of Illinois UrbanaChampaign, Urbana, IL 61801

§Department of Chemical Engineering, University of Florida, Gainesville, FL 32611

MATLAB Phase Sensitive Detection Code 
$\% \% \% \%$ Resampling to single period $\% \% \% \% \%$

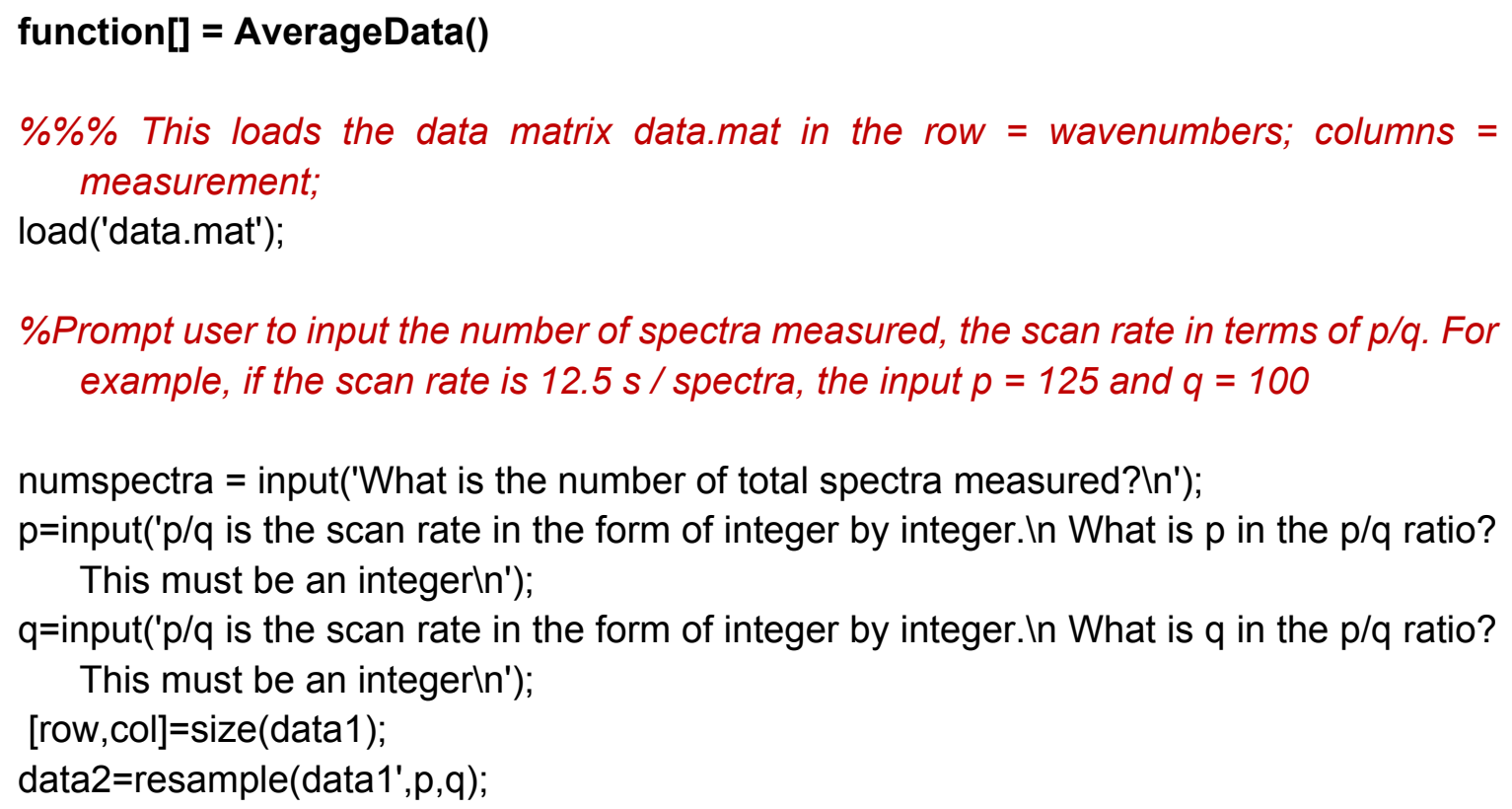




\title{
$\% \% \% \%$ Phase demodulation conversion $\% \% \% \% \%$
}

\section{function[] = PSD_FrequencyDomain()}

\author{
load CombinedAbsorbance;
}

$\% \% \%$ You can change this increment to change number of columns the final $\% \% \%$ MESPSD program writes to the EXCEL file (i.e., ph_incr=10 would write $\% \% \%$ every 10 th angle and give you 36 spectra)

ph_incr=1; \%the increments of the phaseshift in degrees $\% \% \%$ ph=ph_incr:ph_incr:360; \%define the phase vector in degrees phi=degtorad(ph); \%converts to radians

[row,col] $=$ size(CombinedAbsorbance); random $($ row, 1$)=0$; CombinedAbsorbance $1=$ [CombinedAbsorbance $]$; [row1,col1] =size(CombinedAbsorbance1);

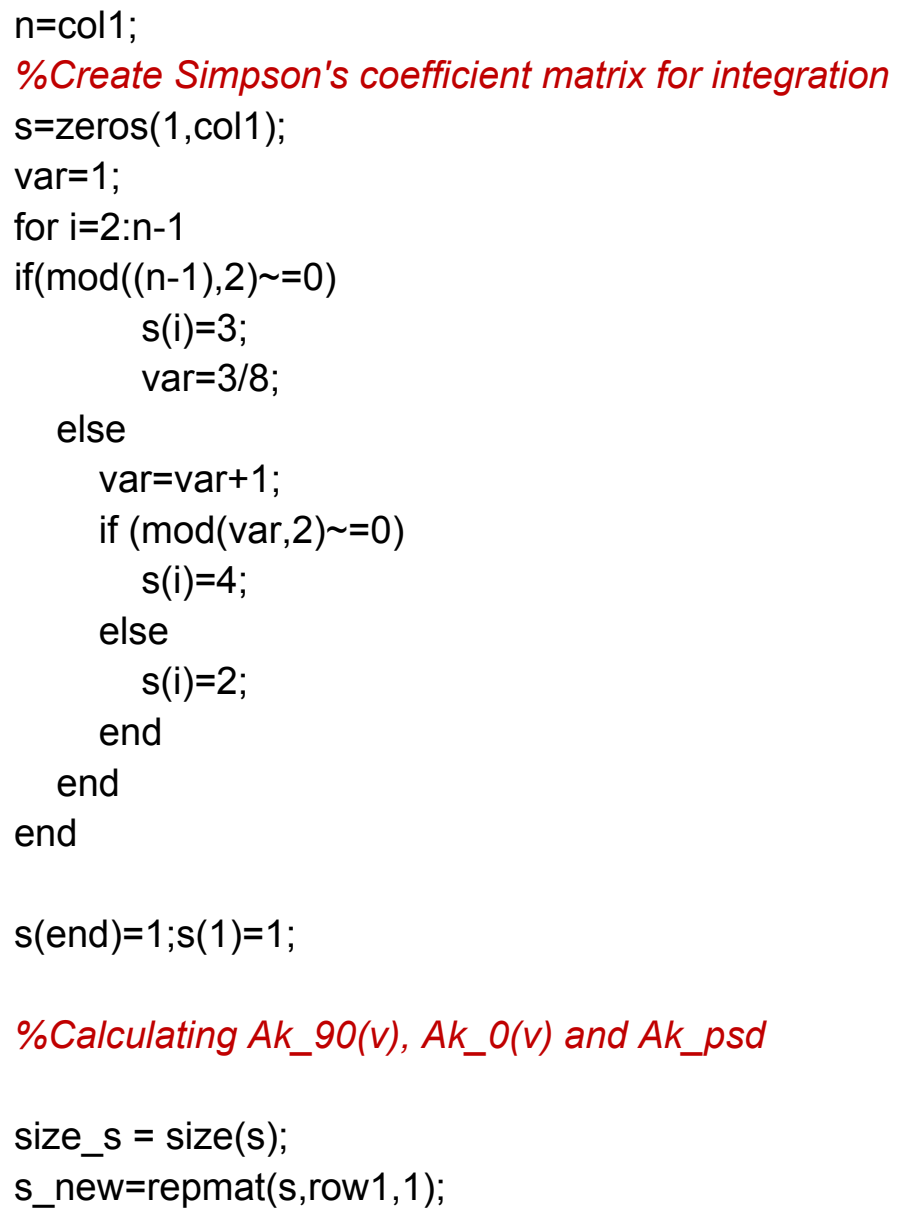




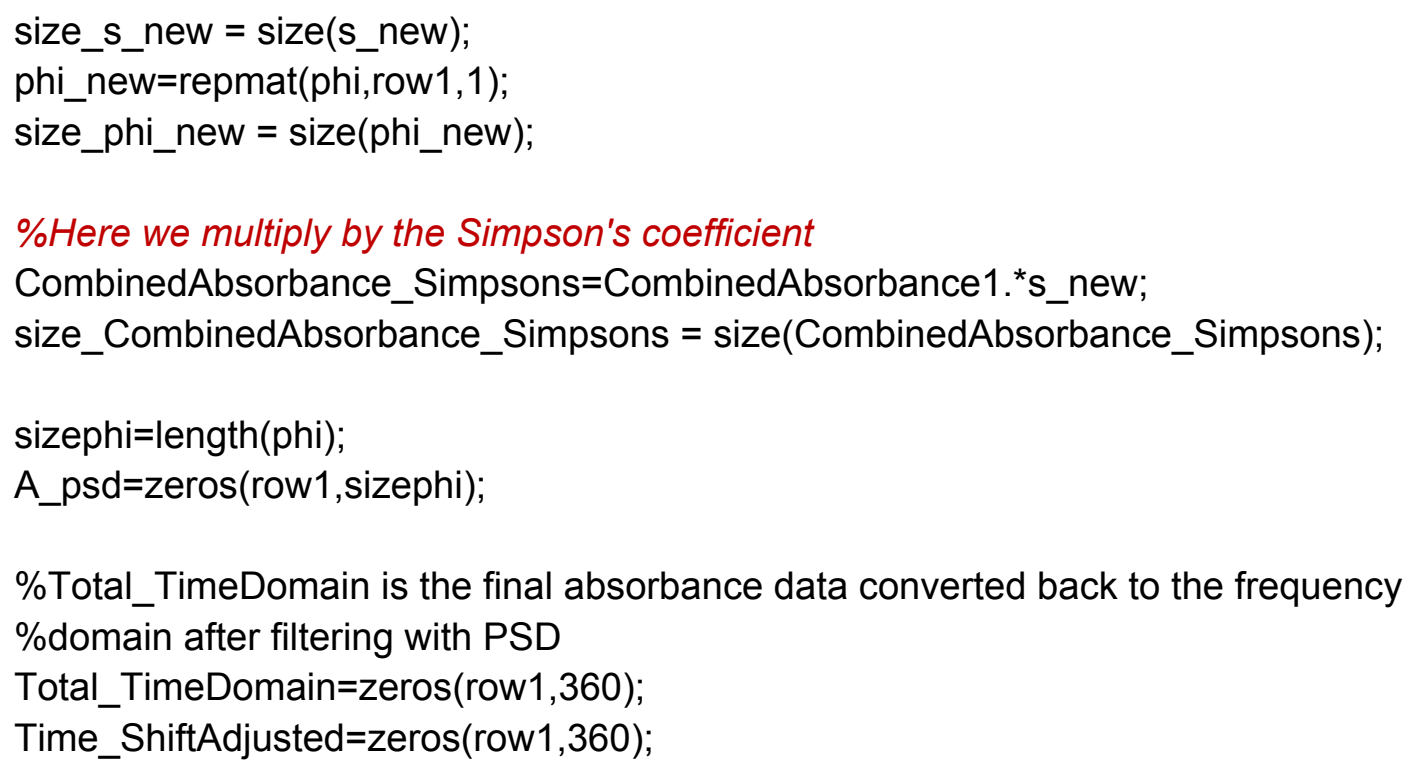

$\% \% \% \%$ This is the number of harmonics it will iterate through. If probing higher harmonics, increase $N$ accordingly. For further information, refer to literature on frequency modulation.

$\mathrm{N}=1$

$\mathrm{T}=\mathrm{col} 1$

$\mathrm{t}=[1: 1: \mathrm{T}]$

$\% \% \%$ This loops through each harmonic individually, coverts the phase data to the frequency domain, which considers the length of a period in seconds, but has removed all the spectral features that do not change at the same frequency as $N$ / timeperiod.

for $k=1: 1: N$

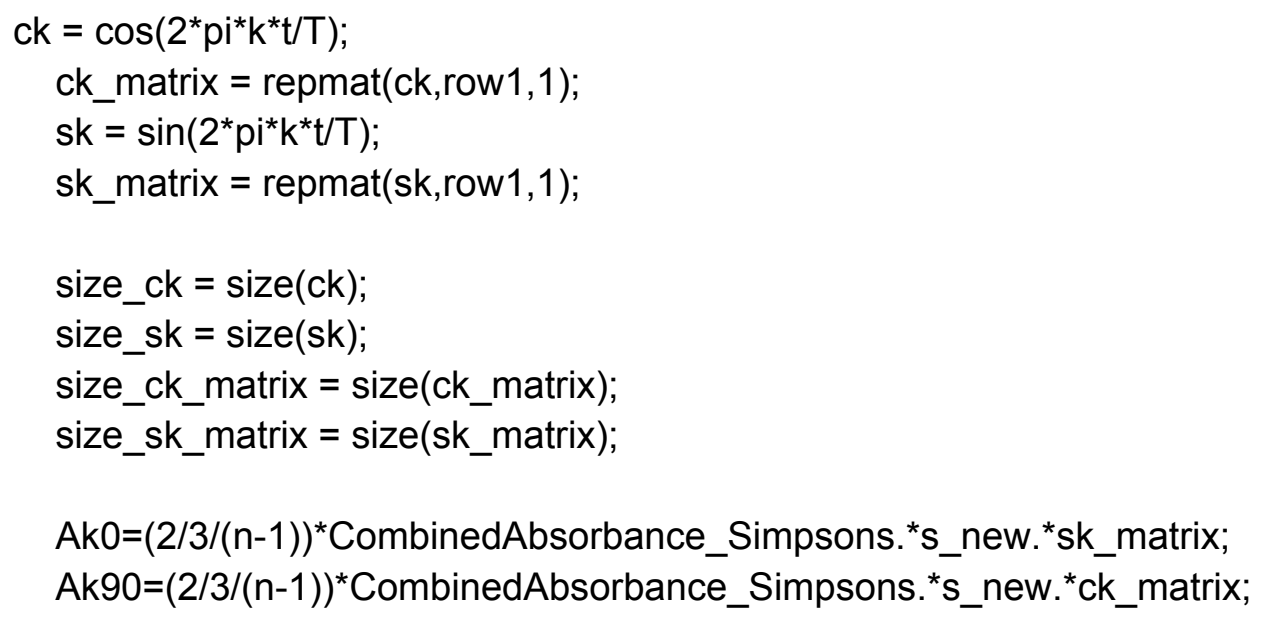




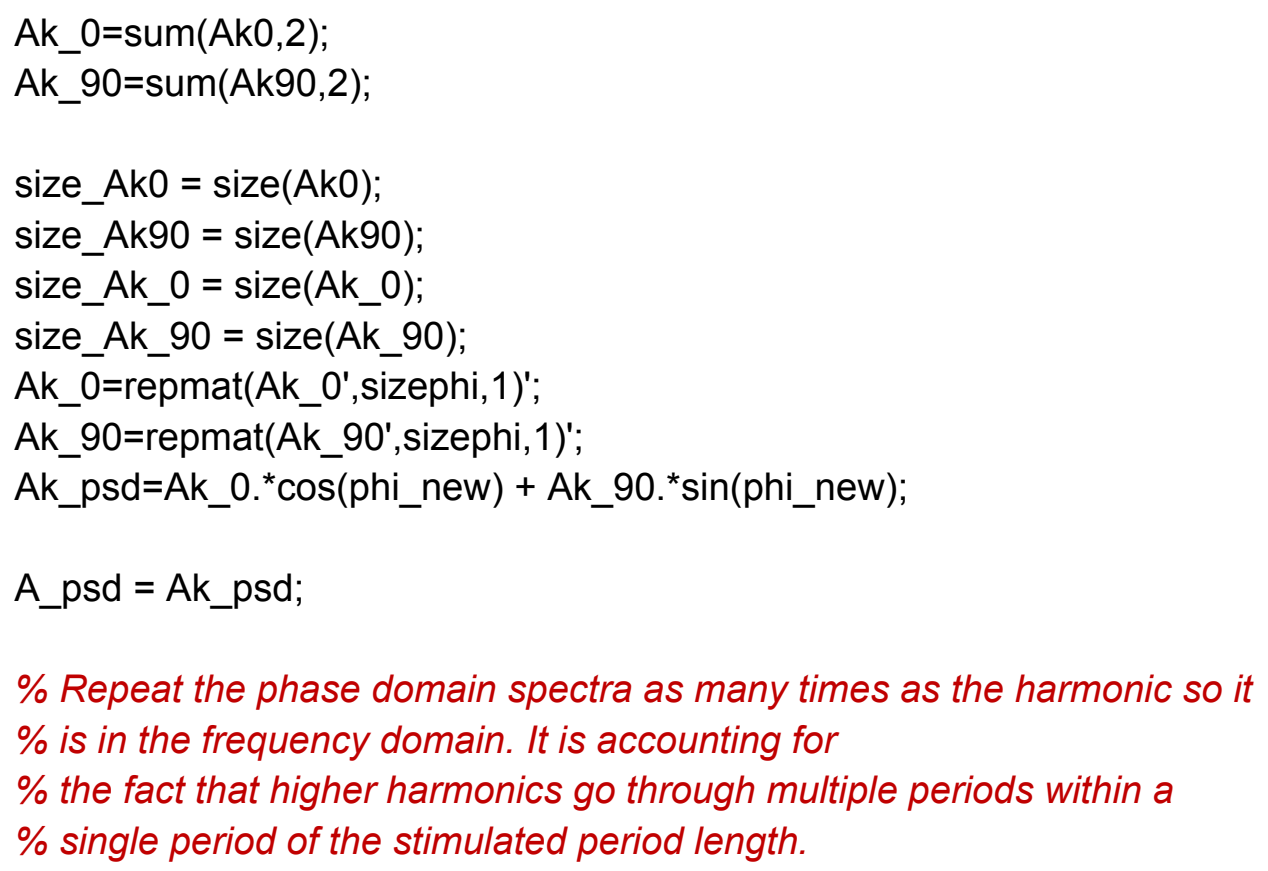

Time_psd = repmat $\left(A \_p s d, 1, k\right)$;

$\%$ Here we resample the frequency domain matrix to be 360 points wide since we just made the phase

$\%$ domain spectra repeat the number of times of the harmonic number

Time_360wide $=$ resample $($ Time_psd',1,k)';

$\%$ Here we are correcting for the fact that the PSD shifts the phase $\%$ shift based on the harmonic value.

Timeshift $=\operatorname{round}\left(360 /\left(4^{*} \mathrm{k}\right)\right)$;

Timeshift_plus1 $=$ Timeshift +1 ;

Timestable $=360$ - Timeshift;

Timestable_plus $1=$ Timestable +1 ;

Time_ShiftAdjusted(:,1:1:Timestable $)=$ Time_360wide(:,Timeshift_plus1:1:360);

Time_ShiftAdjusted(:,Timestable_plus $1: 1: 360)=$ Time_360wide(:,1:1:Timeshift);

$\%$ Add the current harmonic to the combined frequency domain matrix. End

Total_TimeDomain $=$ Total_TimeDomain + Time_ShiftAdjusted. ${ }^{*}-1$;

save Time_PhaseDomain; 
$\% \% H e r e$ we do not write to a specific file like .xlsx or .csv, but this is the appropriate placement for that step to be added if desired.

end

Subsequent identification of kinetically distinct components by singular value decomposition and multivariate curve resolution was performed by feeding the output of this code to the MCR-ALS program described by citation 33 in the main text (Jaumot, J.; de Juan, A.; Tauler, R. Chemometr Intell Lab 2015, 140, 1. 